\title{
Avaliação do Uso de Plantas Medicinais na Academia da Saúde do Município de Rio de Contas/BA
}

\author{
Edlucio Souza Ramos ${ }^{1}$; Rodrigo Santos Damascena ${ }^{2}$
}

\begin{abstract}
Resumo: Na contemporaneidade a utilização de plantas medicinais como escolha terapêutica está se baseando em uma demanda de público cada vez mais abrangente. O uso de plantas medicinais com intuito de tratar várias doenças é algo explícito que faz parte da cultura humana. O objetivo dessa pesquisa foi avaliar a utilização de plantas medicinais pelos usuários da Academia da Saúde localizada no município de Rio de Contas/Ba. A pesquisa foi do tipo quantitativa, envolvendo trabalho de campo, apresentando como instrumento de coleta de dados um questionário individualizado. O estudo analisou também questões culturais e econômicas da população, sendo importante o incentivo dessa temática etnobotânica e etnofarmacológica para o aumento de informações sobre as plantas medicinais. Foram entrevistadas 50 pessoas as quais citaram as espécies mais utilizadas, forma de preparo, parte da planta e indicação de uso. As espécies mais citadas apresentaram maior indicação como calmante, doenças crônicas, problemas digestivos, respiratórios e neurológicos. Entretanto, os resultados mostraram a necessidade de um profissional qualificado que forneça informações sobre a utilização.
\end{abstract}

Palavras-chave: plantas medicinais; terapias alternativas; academia da saúde.

\section{Evaluation of the use of Medicinal Plants in the Health Academy of the Municipality of Rio de Contas / BA}

\begin{abstract}
In contemporary times the use of medicinal plants as a therapeutic choice is being based on an increasingly broad public demand. The use of medicinal plants in order to treat various diseases is something explicit that is part of human culture. The objective of this research was to evaluate the use of medicinal plants by the users of the Health Academy located in the city of Rio de Contas / Ba. The research was of the quantitative type, involving field work, presenting as an instrument of data collection an individualized questionnaire. The study also analyzed cultural and economic issues of the population, and it is important to encourage this ethnobotanical and ethno-pharmacological theme to increase information about medicinal plants. Fifty people were interviewed, which mentioned the most used species, preparation form, part of the plant and indication of use. The most cited species presented greater indication as calming, chronic diseases, digestive, respiratory and neurological problems. However, the results showed a need for a qualified professional to provide information on use.
\end{abstract}

Keywords: medicinal plants, alternative therapies, health academy.

\footnotetext{
${ }^{1}$ Graduando em Farmácia pela Faculdade Independente do Nordeste - FAINOR. edinho99_sr@ hotmail.com

${ }^{2}$ Graduação em Ciências Farmacêuticas pela Universidade Estadual de Feira de Santana. Mestre em Saúde Pública pela Fiocruz. Especialista em Microbiologia Clínica. Especialista em Saúde Pública com Ênfase em PSF. Especialista em Gestão da Assistência Farmacêutica. Especialista em Farmácia Clínica e Hospitalar.
} 


\section{Introdução}

Na contemporaneidade a utilização de plantas medicinais como escolha terapêutica está se baseando em uma demanda de público cada vez mais abrangente. Dessa forma, a medida que ocorrem os avanços tecnológicos e consequentes pesquisas sobre o tema, a população tem obtido mais conhecimentos para aprimorar essa terapia (SALTOS et al., 2016).

Este aumento faz com que pesquisas e estudos apresentem um maior comprometimento em aprovisionar dados em relação ao princípio de produtividade das plantas e preparo dos fármacos. Estas vêm fornecendo ao longo dos anos uma grande contribuição para o desenvolvimento de diversos medicamentos, além do seu emprego na medicina popular com alvos terapêuticos (ATANASOV et al., 2015).

Segundo RAJ et al. (2018), o uso de plantas medicinais com intuito de tratar várias doenças é algo explícito que faz parte da cultura humana. O conhecimento etnomedicinal é um recurso antigo valioso para o cuidado da saúde das pessoas, sendo, portanto, um saber milenar para futura sociedade no uso sustentável e sua conservação. A informação crucial sobre o uso dessas plantas é ampla, sendo na maioria das vezes a única solução para o tratamento da saúde de sociedades rurais de países em desenvolvimento (LALEYE et al., 2015).

Em 1986, ocorreu no Brasil a $8^{\text {a }}$ Conferência Nacional de Saúde, onde instituiu temas relacionados a saúde da população e dentre eles o uso de plantas medicinais na atenção básica. Dentro deste contexto, foi relatado debates sobre a necessidade de introduzir práticas tradicionais para a cura no atendimento público de saúde, aprimorando e valorizando os saberes tradicionais das pessoas a este tipo de terapia (ROSA; CÂMARA; BÉRIA, 2011).

Através do Decreto $n^{\circ} 5.813$ de 2006, foi aprovada a Política Nacional de Plantas Medicinais e Fitoterápicos (PNPMF) com o intuito de garantir a qualidade, eficiência e eficácia dessas plantas desde o seu cultivo até a sua dispensação (MATSUCHITA; MATSUCHITA, 2015). A PNPMF visa garantir aos brasileiros o acesso seguro e uso racional dessas plantas e fitoterápicos para que seja utilizada de forma sustentável, não provocando assim desgaste na biodiversidade do planeta (ANTONIO, 2013).

Nota-se que na última década ocorreram avanços em relação ao uso de práticas terapêuticas alternativas, tendo suporte por políticas no contexto do Sistema Único de Saúde (SUS), apresentando como um dos principais enfoques o uso de fitoterápicos e de plantas medicinais (ZENI et al., 2017). Dessa forma, foi estabelecido a Política Nacional de Práticas

76 Id on Line Rev. Mult. Psic. V.12, N. 42, Supl. 1, p. 75-84, 2018 - ISSN 1981-1179

Edição eletrônica em http://idonline.emnuvens.com.br/id 
Integrativas e Complementares (PNPIC) que engloba: a medicina antroposófica, homeopatia, medicina tradicional chinesa/acupuntura, termalismo, plantas medicinais e fitoterapia (SÜSSKIND et al., 2011).

Tendo em vista que o Brasil condiz com os interesses e busca pelas práticas não convencionais em saúde (PNCS), a publicação da PNPIC em 2006, teve como um dos objetivos principais conceder a população serviços relacionados a plantas medicinais e fitoterápicos. Práticas essas que tem como interesse passar para o usuário informações da ação terapêutica, efeitos tóxicos, indicação e contraindicação, forma correta do preparo e cultivo das plantas (IBIAPINA et al., 2014).

Segundo a Portaria do Ministério da Saúde, o Programa Academia da Saúde consiste em espaços públicos para o desenvolvimento das atividades, tendo como principal propósito contribuir para a promoção da saúde, instituindo um quadro de pessoas qualificadas para melhor orientá-los sobre práticas corporais e atividades físicas, apresentando modos de vida saudáveis e de lazer para essas pessoas (BRASIL, 2011).

Boa parte da população que frequentam esses estabelecimentos são de pessoas idosas e adultos. Dessa forma, a Organização Mundial de Saúde (OMS) estima que cerca de 80\% da população faz ou já fizeram uso de plantas com finalidade terapêutica, apresentando uma maior parcela dessas pessoas, onde os mesmos acreditam que essas ervas estão livres de provocar qualquer efeito colateral (CARNEIRO et al., 2014).

A etnofarmacologia irá obter estudos relacionados as interações das pessoas com as plantas. A mesma terá a missão de melhor explicar e lapidar sobre os recursos vegetais em relação a população (YEUNG; HEINRICH; ATANASOV, 2018).

Diante dos fatores sociais e econômicos, o elevado custo dos fármacos industrializados, os obstáculos enfrentados pelas pessoas em receber assistência médica, faz com que ocorra o aumento dos recursos medicinais utilizando plantas (BELTRÁN et al., 2014). Perante este contexto o uso racional das plantas medicinais tem como alvo garantir melhores condições de saúde, abrindo um acervo de informações sobre as ervas medicinais (CHELLAPPANDIAN et al., 2018).

Portanto, este trabalho teve por objetivo realizar o levantamento do consumo de plantas medicinais por usuários da Academia da Saúde no município de Rio de Contas/Ba. Esses dados 
poderão ser utilizados futuramente para subsidiar ações de educação em saúde para esta população.

\section{Materiais e Métodos}

Esta pesquisa trata-se de um estudo epidemiológico observacional, tipo seccional, descritivo e quantitativo dos dados colhidos (ZENI et al., 2017). Foi realizada na Academia da Saúde do município de Rio de contas, ao Sul da Chapada Diamantina, nas coordenadas (13 $34^{\circ}$ 44" de Latitude Sul e 41 48' 41" de Longitude Oeste), com altitude média de 994m. Segundo dados do último censo IBGE (2010) a população em 2010 foi de 13.007 habitantes, correspondendo a uma densidade demográfica de 12,23 habitantes $/ \mathrm{km}^{2}$.

O período de execução da pesquisa ocorreu no mês de agosto de 2018 utilizando entrevistas com questionários de atributos quantitativos, cujo critério para escolha foi à disponibilidade dos participantes em responder as perguntas. Foram abordados aspectos socioeconômicos e culturais, observando os dados quanto a finalidade do seu uso, quais as partes, o modo de utilização das plantas e a relação dessas plantas com os entrevistados.

Como critério de inclusão, foram entrevistadas pessoas maiores de 18 anos que frequentam a Academia da Saúde e que concordaram em assinar o Termo de Consentimento Livre e Esclarecido (TCLE), de acordo com a resolução 466/12, 510/16 e 580/18 do Ministério da Saúde.

Ao final da pesquisa os dados foram analisados através de estatística descritiva e apresentados na forma de frequência e porcentagem para cada variável analisada, sendo ilustrados através de tabelas.

\section{Resultados e Discussão}

Foram entrevistados 50 indivíduos que frequentam a Academia da Saúde do município de Rio de Contas/Ba, destes 48 foram mulheres (96,00\%), com média de idade de 50,8 anos e um desvio padrão de 13,04. A maioria $(50,00 \%)$ dos entrevistados declarou-se cor parda, 
casado $(54,00 \%)$ e $(72,00 \%)$ com renda mensal do grupo familiar de 01 a 03 salários mínimos. Em relação aos estudos, o ensino médio completo obteve o maior percentual de escolaridade dos usuários $(52,00 \%)$ (Tabela1).

Tabela 1. Caracterização socioeconômica dos usuários atendidos pela Academia da Saúde em Rio de Contas (BA).

\begin{tabular}{|c|c|c|}
\hline Variável & $\mathbf{N}^{0}$ de entrevistados & $(\%)$ \\
\hline \multicolumn{3}{|l|}{ Sexo } \\
\hline Masculino & 2 & 4,00 \\
\hline Feminino & 48 & 96,00 \\
\hline Total & 50 & 100,00 \\
\hline \multicolumn{3}{|l|}{ Faixa Etária } \\
\hline $20-30$ & 6 & 12,00 \\
\hline $31-40$ & 2 & 4,00 \\
\hline $41-50$ & 13 & 26,00 \\
\hline $51-60$ & 16 & 32,00 \\
\hline $61-70$ & 12 & 24,00 \\
\hline $71-80$ & 1 & 2,00 \\
\hline Total & 50 & 100,00 \\
\hline \multicolumn{3}{|l|}{ Cor autodeclarada } \\
\hline Branco (a) & 9 & 18,00 \\
\hline Preto (a) & 15 & 30,00 \\
\hline Pardo (a) & 25 & 50,00 \\
\hline Amarelo (a) & 1 & 2,00 \\
\hline Indígena & 0 & 0,00 \\
\hline Total & 50 & 100,00 \\
\hline \multicolumn{3}{|l|}{ Estado Civil } \\
\hline Solteiro (a) & 7 & 14,00 \\
\hline Casado (a) & 27 & 54,00 \\
\hline Viúvo (a) & 7 & 14,00 \\
\hline Separado (a) & 9 & 18,00 \\
\hline Total & 50 & 100,00 \\
\hline \multicolumn{3}{|l|}{ Renda Mensal } \\
\hline Menos de 1 salário mínimo & 11 & 22,00 \\
\hline De 1 a 3 salários mínimos & 36 & 72,00 \\
\hline De 3 a 6 salários mínimos & 1 & 2,00 \\
\hline De 6 a 9 salários mínimos & 0 & 0,00 \\
\hline Mais de 9 salários mínimos & 2 & 4,00 \\
\hline Total & 50 & 100,00 \\
\hline \multicolumn{3}{|l|}{ Escolaridade } \\
\hline Não estudou & 0 & 0,00 \\
\hline Ensino Fundamental & 18 & 36,00 \\
\hline Ensino Médio Incompleto & 1 & 2,00 \\
\hline Ensino Médio Completo & 26 & 52,00 \\
\hline Ensino Superior Incompleto & 1 & 2,00 \\
\hline Ensino Superior Completo & 2 & 4,00 \\
\hline Pós-graduação & 1 & 2,00 \\
\hline Não sei & 1 & 2,00 \\
\hline Total & 50 & 100,00 \\
\hline
\end{tabular}

Fonte: Elaborado pelos autores, 2018.

O presente estudo avaliou a utilização de plantas medicinais e as variáveis que levaram ao uso das mesmas pelos frequentadores da Academia da Saúde. Os dados coletados podem 
contribuir como informações relevantes aos gestores municipais e à comunidade como um conjunto de entendimento de terapia praticadas pela população.

Os dados socioeconômicos da amostra demostram semelhança a outras pesquisas realizadas em Programas do Ministério da Saúde no Brasil, tendo como predomínio de pessoas adultas, do sexo feminino pertencentes a classe média social (TOMASI, 2011).

Verificou-se que $86 \%$ dos usuários entrevistados utilizam plantas medicinais como uma terapia alternativa para tratar as afecções. Neste sentido, Carvalho et al. (2013) informaram dados semelhantes, destacando que as plantas medicinais apresentam-se uma alternativa pelo o seu baixo custo e sua eficiência terapêutica.

Tabela 2. Relação das 10 plantas mais citadas, frequência e indicação de uso medicinal relatadas pelos usuários da Academia da Saúde em Rio de Contas (BA).

\begin{tabular}{llll}
\hline Nome popular & $N^{\mathbf{0}}$ & $\%$ & Indicações populares \\
\hline Erva-cidreira & 32 & 15,76 & "Calmante, doenças crônica, problemas digestivos, respiratório e neurológicos" \\
Erva-doce & 31 & 15,27 & "Calmante, doenças crônica, problemas digestivos, respiratório e neurológicos" \\
Capim-Santo & 26 & 12,81 & "Calmante, doenças crônica, problemas digestivos, respiratório e neurológicos" \\
Hortelã & 16 & 7,88 & "Calmante, doenças crônica, problemas digestivos, respiratório e neurológicos" \\
Camomila & 8 & 3,94 & "Calmante, doenças crônica, problemas digestivos, respiratório e neurológicos" \\
Folha de laranjeira & 6 & 2,96 & "Problema respiratório, problemas digestivos" \\
Gengibre & 6 & 2,96 & "Problema digestivo, Problema respiratório" \\
Amora & 5 & 2,43 & "Problema respiratório, problemas digestivos" \\
Cravo & 5 & 2,43 & "Problema digestivos, Problema respiratório" \\
Salsa & 5 & 2,43 & "Problema digestivos" \\
Outros & 63 & 31,04 & ------------ \\
Total & 203 & 100,00 & \\
\hline
\end{tabular}

Fonte: Elaborado pelos autores, 2018.

O ato de consumir plantas medicinais foi descrito em um total de 203 vezes pelos entrevistados. As três espécies com maior número de citações foi a erva-cidreira $(15,76 \%)$, em seguida a erva-doce $(15,27 \%)$ e capim-santo $(12,81 \%)$. Neste estudo, foi citado pelos usuários 51 tipos de plantas, sendo que as 10 mais mencionadas estão expostas na tabela 2.

De acordo com as informações coletadas, entre as afecções tratadas com maior frequência destacam-se os problemas digestivos $(54,17 \%)$, problemas respiratórios $(25,00 \%)$ e problemas neurológicos (8,33\%). Zeni e Bosio (2011), observaram resultados divergentes em relação aos problemas digestivos e respiratórios. Além disso, eles enfatizam que entre as 
enfermidades mais comuns tratadas nas Unidades de Saúde, as doenças do aparelho circulatório apresentam maior periodicidade.

Uma grande parcela das pessoas entrevistadas, informaram que as plantas utilizadas são de produção própria $(37,21 \%)$, ou seja, coletadas no próprio quintal, com vizinhos, amigos e familiares $(30,23 \%)$, compra em supermercados ou farmácias $(20,93 \%)$, feiras livres $(11,63 \%)$. Estudos realizados por Henrique et al. (2014) confirmaram resultados similares indicando que $51 \%$ dos entrevistados usam plantas cultivadas em sua própria residência.

A forma de aquisição dessas plantas pode trazer alguns riscos existentes, como a contaminação por fatores extrínsecos, sendo eles causados por parasitas que podem provocar doença infeciosas, o contágio de pesticidas e metais pesados, além da adição de substâncias com a finalidade de prolongar o efeito dos vegetais. Outro risco seria a possibilidade de toxicidade a planta. Esses ricos podem ser potencializados quando as ervas são obtidas em vendas de produtos naturais, feiras livres ou mercados públicos (NASCIMENTO et al., 2013).

Já relacionado ao benefício, é possível destacar que existe a facilidade do acesso, além de não haver custo de aquisição quando a planta é cultivada em sua própria residência, isso também faz com que o risco de contaminação por substâncias químicas seja minimizado (GADELHA et al., 2013)

Observou-se que de um total de 43 pessoas que usam plantas medicinais, $(37,86 \%)$ tem preferência pela folha, sendo a mesma, a forma de obtenção mais utilizada. Já para o seu preparo constatou que a infusão obteve uma maior prevalência com $(47,50 \%)$, em seguida a decocção com (18,75\%). Zeni e Bosio (2011) comprovam em seus estudos uma constante semelhante dos resultados apresentados.

Vale ressaltar, que das 43 pessoas que participaram dessa pesquisa e relataram que utilizam plantas medicinais, 17 estão entre 20 e 50 anos e 26 são maiores de 50 anos. A média de idade daqueles que fazem uso de alguma forma foi de 51,0 anos, e os que não usam foi 49,5 anos. Esse comportamento também foi observado por David e Pasa (2015), onde existe uma diferença significativa quando realiza a relação da idade com a utilização dessas plantas, ou seja, pessoas idosas utilizam com maior frequência. Essa diferença pode estar ligada com a cultura, seja ela familiar ou social.

Entre as 10 plantas mais citadas pelos os usuários, quatro fazem parte lista de 71 plantas mencionadas na Relação Nacional de Plantas Medicinais de Interesse ao SUS RENISUS 
(BRASIL, 2009). Devido a Academia da Saúde ser um programa público, essa comparação de utilização dos vegetais com as plantas presentes no RENISUS, se deve por ser uma política pública com finalidade de orientar estudos e pesquisas para a população fazer uso com segurança e eficácia, sendo assim é ideal que seja estimulado o uso delas.

No decorrer do presente estudo, percebeu-se que a utilização de plantas medicinais pelos usuários da academia da saúde em Rio de Contas/Ba se faz muito presente, representando um importante suporte das condições de saúde dessa população. Isso faz com que os costumes e cultura de um saber local seja preservado e utilizado.

A Organização Mundial da Saúde (OMS), vem proporcionando o uso de plantas medicinais com incentivo e valorização de práticas terapêuticas tradicionais para atender algumas necessidades de saúde da população, podendo contribuir para os usuários do sistema público de saúde uma garantia do uso racional (BRUNING; MOSEGUI; VIANNA, 2012).

\section{Considerações Finais}

O uso de plantas medicinais é um recurso antigo e de grande importância para a população. A preservação da biodiversidade, a busca por alternativas e a troca de experiências são temas primordiais para promover a qualidade de vida dos usuários. A Política Nacional de Práticas Integrativas e Complementares tem como propósito passar para a sociedade informações da ação terapêutica, efeitos tóxicos, indicação e contraindicação, forma correta do preparo e cultivo das plantas. Os dados da referida pesquisa podem ser aprofundados com o sentido de criar políticas públicas que estimulem o uso desse tipo de terapia, pois é algo que está presente na população, mas as vezes sem o acompanhamento de um profissional habilitado que tenha o intuído de orientar a forma correta do uso, da identificação, levando em conta o uso racional dos mesmos. 


\section{Referências}

ANTONIO, G. D. FITOTERAPIA NA ATENÇÃO PRIMÁRIA À SAÚDE Interação de saberes e práticas de cuidado. Florianópolis. 2013.

ATANASOV, A. G. et al. Discovery and resupply of pharmacologically active plant-derived natural products: A review. Biotechnology Advances, v. 33, n. 8, p. 1582-1614, 2015.

BELTRÁN-RODRÍGUEZ, L. et al. Factors affecting ethnobotanical knowledge in a mestizo community of the Sierra de Huautla Biosphere Reserve, Mexico. Journal of Ethnobiology and Ethnomedicine, v. 10, n. 1, 2014.

BRASIL. MS elabora Relação de Plantas Medicinais de Interesse ao SUS 06/03/2009. Agência Saúde, p. 3-5, 2009.

BRASIL. Ministério da Saúde Portaria N 719 , DE 07 DE ABRIL DE 2011.

BRUNING, M. C. R.; MOSEGUI, G. B. G.; VIANNA, C. M. DE M. A utilização da fitoterapia e de plantas medicinais em unidades básicas de saúde nos municípios de Cascavel e Foz do Iguaçu - Paraná: a visão dos profissionais de saúde. Ciência \& Saúde Coletiva, v. 17, n. 10, p. 2675-2685, 2012.

CARNEIRO, F. M. et al. Tendências Dos Estudos Com Plantas Medicinais No Brasil. Revista Sapiência: sociedade, saberes e práticas educacionais, v. 3, n. 2, p. 44-75, 2014.

CARVALHO, J. S. B. DE et al. Uso popular das plantas medicinais na comunidade da Várzea, Garanhuns-PE. Revista de Biologia e Ciências da Terra, v. 13, n. 2, p. 58-65, 2013.

CHELLAPPANDIAN, M. et al. Traditionally practiced medicinal plant extracts inhibit the ergosterol biosynthesis of clinically isolated dermatophytic pathogens. Journal de Mycologie Medicale, v. 28, n. 1, p. 143-149, 2018.

DE DAVID, M.; PASA, M. C. As plantas medicinais e a etnobotânica em Várzea Grande, MT, Brasil. Interações (Campo Grande), v. 16, n. 1, p. 97-108, 2015.

GADELHA, C. S. et al. Estudo bibliográfico sobre o uso das plantas medicinais e fitoterápicos no Brasil Bibliographical. Revista Verde (Mossoró - RN), v. 8, n. 5, p. 208 - 212, dezembro, 2013

HENRIQUE, B. et al. Importância do conhecimento tradicional no uso de plantas medicinais em Buritis , MG , Brasil. Revista do Centro de Ciências Naturais e Exatas - UFSM, 2014.

IBGE Cidades. Disponível em: https://cidades.ibge.gov.br/brasil/ba/rio-de-contas/panorama. Acesso em 20 set. 2018

IBIAPINA, W. V. et al. INSERÇÃO DA FITOTERAPIA NA ATENÇÃO PRIMÁRIA AOS USUÁRIOS DO SUS. v. 12, n. 1, p. 58-68, 2014.

LALEYE, F. O. A. et al. Diversity, knowledge, and use of plants in traditional treatment of diabetes in the Republic of Benin. Ethnobotany Research and Applications, v. 14, n. October, p. 231-258, 2015. 
MATSUCHITA, H. L. P.; MATSUCHITA, A. S. P. A Contextualização da Fitoterapia na Saúde Pública The Contextualization of Phytotherapy in Public Health. Uniciências, v. 19, n. 1, p. 86-92, 2015.

NASCIMENTO, W. M. C. et al. Plantas medicinais e sua utilização pelas comunidades do município de sobral, ceará. S A N A R E, Sobral, V.12, n.1, p. 46-53, jan./jun. - 2013

RAJ, A. J. et al. Indigenous uses of ethnomedicinal plants among forest-dependent communities of Northern Bengal, India. Journal of Ethnobiology and Ethnomedicine, v. 14, n. 1, p. 1-28, 2018.

ROSA, C. DA; CÂMARA, S. G.; BÉRIA, J. U. Representações e intenção de uso da fitoterapia na atenção básica à saúde. Ciência \& Saúde Coletiva, v. 16, n. 1, p. 311-318, 2011.

SALTOS, R. V. A. et al. The use of medicinal plants by rural populations of the Pastaza province in the Ecuadorian Amazon. Acta Amazonica, v. 46, n. 4, p. 355-366, 2016.

SÜSSKIND, M. et al. Adverse drug reactions in a complementary medicine hospital: A prospective, intensified surveillance study. Evidence-based Complementary and Alternative Medicine, v. 2012, 2011.

TOMASI, E. ET AL. Características da utilização de serviços de Atenção Básica à Saúde nas regiões Sul e Nordeste do Brasil: diferenças por modelo de atenção. Ciencias \& Saúde Coletiva, p. 4395-4404, 2011.

YEUNG, A. W. K.; HEINRICH, M.; ATANASOV, A. G. Ethnopharmacology-A bibliometric analysis of a field of research meandering between medicine and food science? Frontiers in Pharmacology, v. 9, n. MAR, 2018.

ZENI, A. L. B. et al. Utilização de plantas medicinais como remédio caseiro na Atenção Primária em Blumenau, Santa Catarina, Brasil. Ciência \& Saúde Coletiva, v. 22, n. 8, p. 2703-2712, 2017.

ZENI, A. L.; BOSIO, F. O uso de plantas medicinais em uma comunidade rural de Mata Atlântica Nova Rússia, SC. Neotropical Biology and Conservation, v. 6, n. 1, p. 55-63, 2011.

\section{Como citar este artigo (Formato ABNT):}

RAMOS, Edlucio Souza; DAMASCENA, Rodrigo Santos. Avaliação do Uso de Plantas Medicinais na Academia da Saúde do Município de Rio de Contas/BA. Id on Line Rev.Mult. Psic., 2018, vol.12, n.42, Supl. 1, p. 75-84. ISSN: 1981-1179.

Recebido: 29/10/2018;

Aceito: 30/10/2018 